\title{
Implementing Quality of Service in Shipboard Power System Design
}

\author{
Dr. Norbert H. Doerry. \\ Naval Sea Systems Command \\ U.S. Navy \\ Washington DC
}

\begin{abstract}
Quality of Service (QOS) is a measure of the ability of the power system to reliably provide loads electrical power with the power continuity required by the loads. QOS recognizes that different loads can tolerate different power interruption durations. This paper reviews QOS concepts as described in IEEE 1662-2008 and IEEE 1709-2010, describes methods for employing QOS in the design of shipboard power systems, and presents modeling and simulation considerations.
\end{abstract}

\section{INTRODUCTION}

Quality of Service (QOS) is a measure of the ability of the power system to reliably provide loads electrical power with the power continuity required by the loads. QOS recognizes that different loads can tolerate different power interruption durations. Recognizing this difference in load needs can result in power systems that cost less, both in acquisition and in operation without negatively impacting the performance of the ship. QOS is also an effective means of ensuring two competing power system alternatives have the same level of power continuity performance. This paper will review QOS concepts as described in IEEE 1662-2008 [1] and IEEE 17092010 [2] and describe methods for employing QOS in the design of shipboard power systems. This paper expands and updates previous work by Doerry and Clayton [3] and Doerry [4]. The views expressed herein are the personal opinions of the authors and are not necessarily the official views of the Department of Defense or any military department thereof.

QOS is measured as a Mean Time Between Service Interruption (MTBSI). A service interruption is a power interruption longer in duration than a load can tolerate. Thus if a resistive heating load with its thermal inertia can tolerate 6 minutes of power interruption, then a 2 minute loss of power would not constitute a service interruption for that load. On the other hand, a computer that can only tolerate $20 \mathrm{~ms}$ of power interruption would experience a service interruption with a 2 minute loss of power. Present day technology can generally support system designs with a MTBSI on the order of 30,000 hours.

QOS is a reliability metric. In designing a system to achieve a specified MTBSI, QOS does not account for survivability events such as battle damage, collisions, fires, or flooding. QOS does account for equipment failures, training, and normal system operation transients.

\author{
Dr. John V. Amy Jr. \\ Office of ASN(RDA) \\ U.S. Navy \\ Washington DC
}

The impact of a given load on the ability of the ship to fulfill its mission should be considered in evaluating whether a load can tolerate an interruption of a given duration. The word "tolerate" implies that the ship's capability is at worst only insignificantly impacted as a result of the power interruption.

As defined in [2], loads can be categorized into one of four categories based primarily on the maximum duration of power interruption each load can tolerate. These durations are compared to two parameters, $\mathrm{t} 1$ and $\mathrm{t} 2$, which are properties of the power system. $\mathrm{t} 1$ is the "Reconfiguration Time" and is defined as the maximum time to reconfigure the distribution system without bringing online additional generators. t1 can be as short as tens of milliseconds for fast acting power electronics based fault detection and isolation systems, and as long as 2 seconds for traditional electromechanical circuit breakers. $\mathrm{t} 2$ is the "Generator Start Time" and is defined as the time to start and bring on line the slowest generator set in the power system. $\mathrm{t} 2$ is generally between 1 and 5 minutes depending on the prime mover technology.

The four QOS load categories are:

1. Un-interruptible Loads -- Un-interruptible loads cannot tolerate a power interruption of duration $\mathrm{t} 1$. The power system is designed to provide these loads with the minimum achievable power interruption with the reliability as defined by the customer specified MTBSI.

2. Short-term Interrupt Loads -- Short-term interrupt loads can tolerate power interruptions of duration $\mathrm{t} 1$ but cannot tolerate power interruptions of duration $t 2$. The power system is designed to provide power to short-term interrupt loads with power interruptions of duration exceeding $\mathrm{t} 1$ less frequently on average than the customer specified MTBSI.

3. Long-term Interrupt Loads -- Long-term interrupt loads can tolerate power interruptions of duration $t 2$. The power system is designed to provide power to long-term interrupt loads with power interruptions of duration exceeding t 2 less frequently on average than the customer specified MTBSI.

4. Exempt Loads -- Exempt loads can also tolerate power interruptions of duration $\mathrm{t} 2$. However, while the power system is designed under normal operation to supply these 
loads, the power system is not explicitly designed to ensure power interruptions of duration exceeding t2 occur less frequently than the customer specified MTBSI. Generally, only a portion of the propulsion load for ships with electric propulsion ${ }^{1}$ integrated with the electric plant are assigned as Exempt Loads to preclude the installation of excess generation capacity.

QOS impacts many aspects of the shipboard power system design that historically have not been explicitly addressed by the power system designer. QOS also provides a convenient metric for ensuring equivalent performance from two technology alternatives to enable a fair comparison of performance.

\section{BACKGROUND}

Historically, many aspects of QOS were implicitly incorporated in the traditional design methods used for shipboard power systems. Examples include:

- The $\mathrm{N}+1$ rule for generator sets ensured sufficient capacity would exist even with one generator set inoperative.

- All loads were treated as short-term interrupt loads. Un-interruptible loads were required to provide their own dedicated Uninterruptible Power Supply (UPS).

- Loads not critical to the ships mission were designated "non-vital" and shed if remaining power generation was not sufficient to service all loads.

Some of the short comings of the traditional design methods include:

- No general guidance existed for sizing energy storage modules.

- Did not consider the total amount of "vital" loads in establishing the sizes of generator sets. Generator sets usually were sized only with regards to the maximum margined electrical load with service life allowance. In some ships, this resulted on occasion in a complete loss of power (dark ship) because of excessive loads and cascading generator set tripping, even after load shedding, following loss of one online generator (remaining generators not sufficient to serve all the vital loads).

- Proliferation of Automatic Bus Transfer (ABT) devices. ABTs employed for both power continuity and survivability reasons without ensuring sufficient

\footnotetext{
${ }^{1}$ An integrated power system uses the same prime movers to generate electricity for propulsion and ship service loads. Because most prime movers have a Mean Time Between Failure (MTBF) significantly lower than the desired MTBSI, electrical systems have historically provided an additional generator set beyond that normally required to satisfy the load $(\mathrm{N}+1$ rule). For propulsion however, a degradation in maximum speed has historically been found acceptable over the added cost of providing additional prime mover capacity. The exempt load accounts for allowing a degradation of maximum speed due to the failure of a prime mover.
}

power generation capacity would exist to serve the loads on the loss of one source of power.

- Proliferation and added maintenance burden of hundreds of uninterruptible power supplies throughout the ship to serve uninterruptible loads.

- Highly conservative load shedding strategy. Load shedding based solely on survivability concerns rather than maximizing ship capability based on the length of power interruption that a load can tolerate.

- Difficulty in determining the impact of a new power distribution system technology. The traditional design methods generally do not enable trading off improved performance in one area with relaxed performance in another area. Therefore, new technologies generally resulted in increased performance at increased cost; an outcome not welcomed by the ship acquisition program manager.

The remainder of the paper will discuss how to incorporate QOS into the design of a power system and how to address the specific shortcomings of the traditional design methods listed above.

The paper assumes all shipboard power systems, including those that do not include electric propulsion (Propulsion Motor Modules), generally adhere to the electrical architecture of the Next Generation Integrated Power System (NGIPS) described initially by Doerry [5]. Progress towards achieving these goals is described by Hoffman et al. [6]. The standard NGIPS module types are:

- Power Generation Modules (PGM) convert fuel to electrical power

- Power Distribution Modules (PDM) consist of switchgear and cabling necessary to distribute the power

- Power Conversion Modules (PCM) convert power from one voltage / frequency to another

- Energy Storage Modules (ESM) store energy provided by and provided to the electrical power system

- Power Loads use electrical power

- Propulsion Motor Modules (PMM) convert electrical power into propulsion for the ship

- Power Control Modules (PCON) consists of software necessary to operate the power system.

These standard module types are employed in four NGIPS Power Generation Architectures and in the Zonal Electrical Distribution Architecture shown in Figs 1-3:

- Low Voltage AC (LVAC) Power Generation is typically employed in ships with less than about 6 MW maximum margined electric load. Normally associated with ships that use mechanical drive instead of electric drive, the electric power is typically generated and distributed as 450 Volts 3 
phase ac power. For ships without substantial DC or un-interruptible loads, the PCM 1A of Fig. 2 may be eliminated and all DC and un-interruptible loads provided via PCM 2A. For more details on PCM 1A and PCM 2A, see [5].

- Medium Voltage AC (MVAC) Power Generation exploits existing technology as used in DDG-1000 and T-AKE 1 for near term ship designs and for ships without a need for high power density. Generally, ships above 25,000 LT displacement do not benefit significantly from power dense equipment. Between 10,000 and 25,000 LT, ship designs should trade off MVAC systems with higher power dense solutions for overall ship affordability and ship suitability. Higher power dense solutions are usually superior for ships below 10,000 LT.

- Medium Voltage DC (MVDC) Power Generation is the long term goal for providing affordable power dense systems. The application of MVDC however, requires the development and standardization of new ways to manage power, assure system stability, detect faults, and isolate faults. New equipment must also be developed and qualified for ship use. Once developed, MVDC promises affordable high power dense power generation systems.

- Medium Voltage Medium Frequency (MVMF) Power Generation As an interim solution until MVDC is developed, MVMF employs existing AC design and production technologies operating above $60 \mathrm{~Hz}$., but below $400 \mathrm{~Hz}$., to gain sufficient power density to enable introduction into small and medium sized surface combatants as well as submarines.

- Zonal Electrical Distribution (ZEDS) takes power produced by one of the Power Generation Architectures, converts it to the form needed by each load, and distributes the power to the loads.

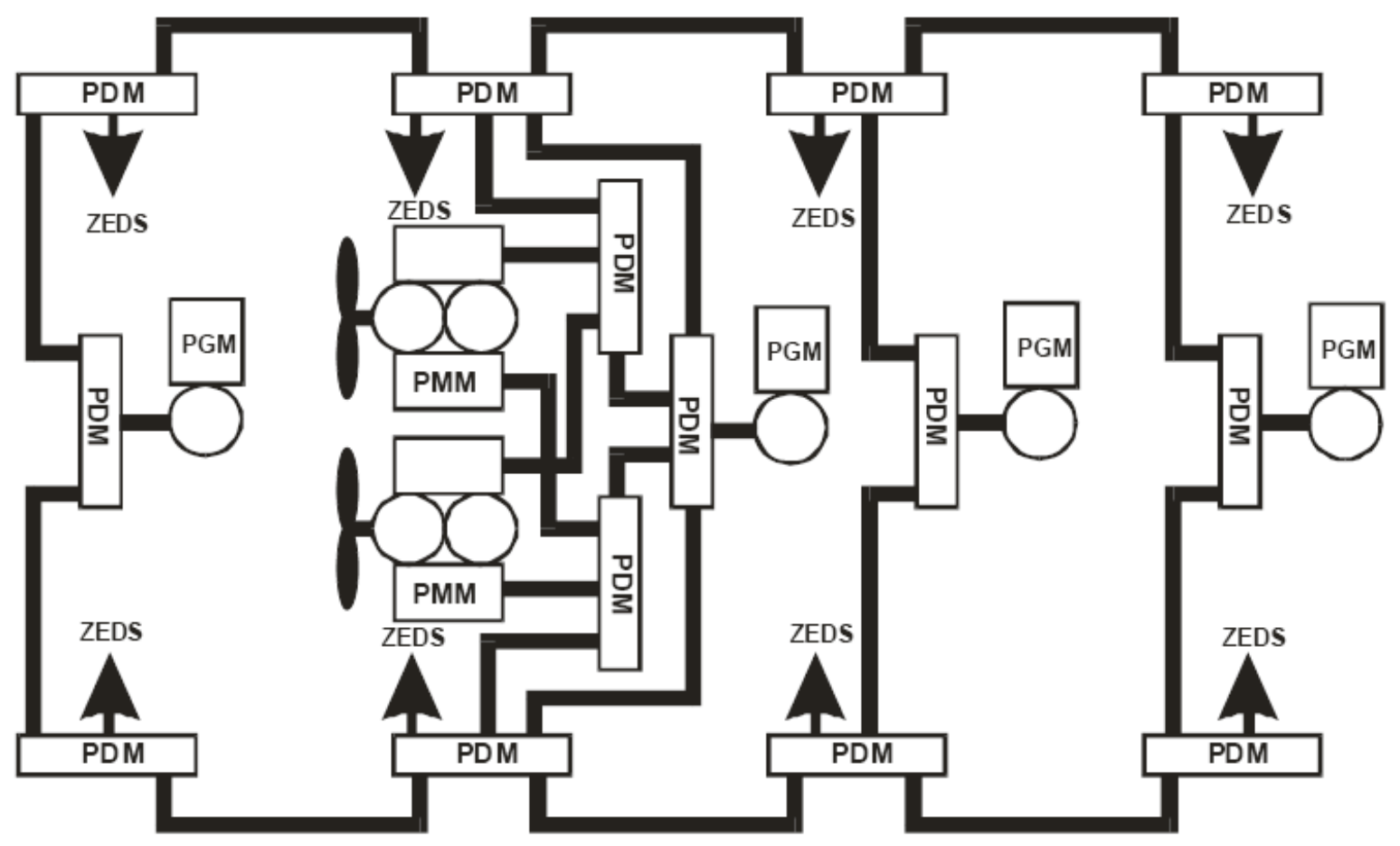

Figure 1. Power Generation Architecture [5] 


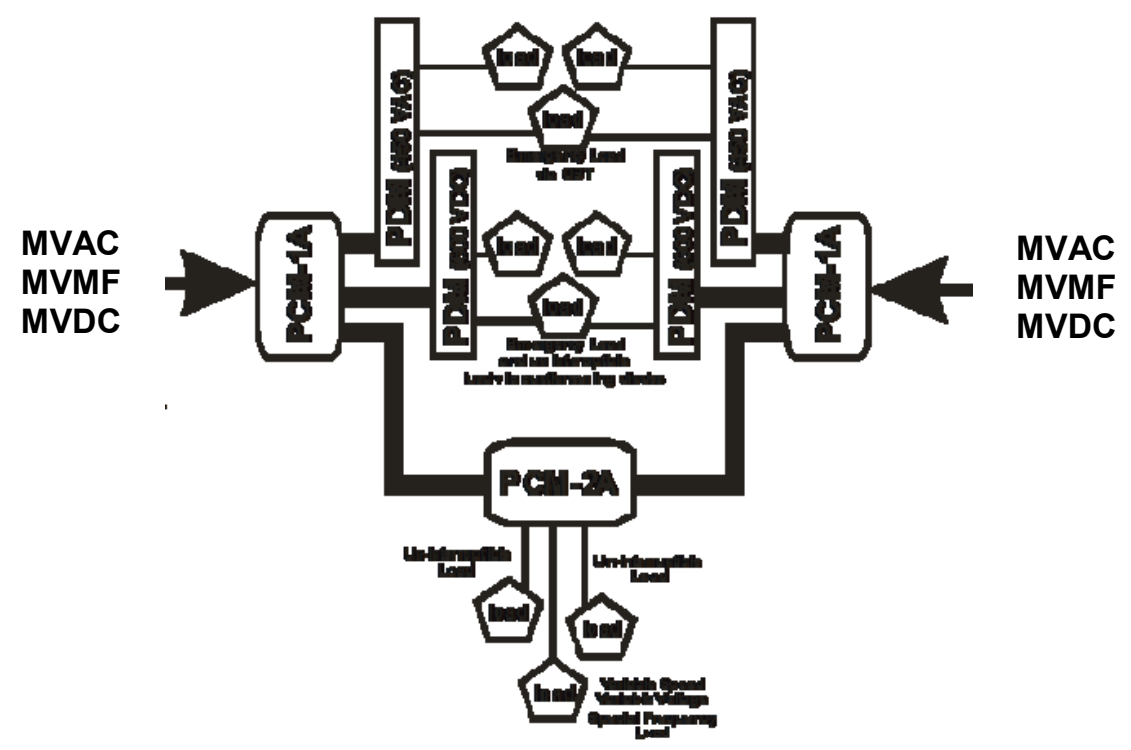

Figure 2. Zonal Electrical Distribution Architecture [5]

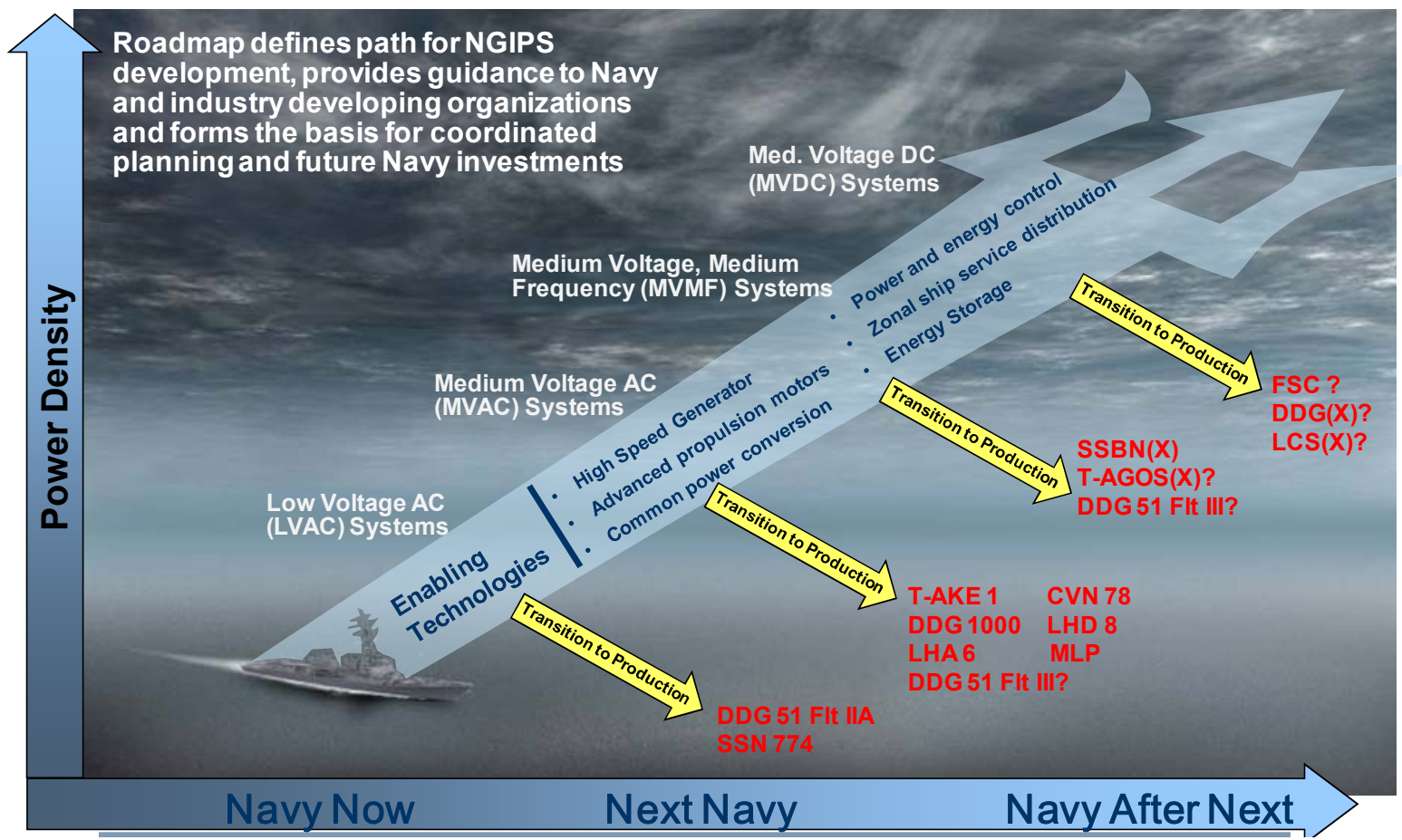

Figure 3. NGIPS Technology Development Roadmap [7]

Energy Storage Modules serve several different functions within a power system. These functions include:

ESM-F1: Isolate un-interruptible loads from short term power interruptions as a consequence of fault detection and system reconfiguration. ESMs fulfilling the $E S M-F 1$ function typically have a rating in the 10's or 100's of $\mathrm{kW}$ and a run time on the order of 10 seconds. The ESM-F1 function typically would not require a large number of chargedischarge cycles (less than 50 per year).
ESM-F2: Provide back-up power on the failure of a PGM or the unanticipated addition of load for un-interruptible and short-term interruptible loads. Also provide standby power until addition PGMs can be brought online for pulse power loads such as rail gun Pulse Forming Networks (PFN), high powered lasers, and advanced radars. ESMs fulfilling the $E S M-F 2$ function typically have a rating in the 100 's of $\mathrm{kW}$ to 10 's of MW and a run time on the order of 1.5 to 6 times t2. The ESM-F2 function typically would not require a large number of charge-discharge cycles (less than 50 per year). 
ESM-F3: Provide emergency starting for a PGM. ESMs fulfilling the $E S M-F 3$ function typically have a rating in the 100 's of $\mathrm{kW}$ and a run time on the order of 15 to 30 minutes. The ESM-F3 function typically would not require a large number of charge-discharge cycles (less than 50 per year).

ESM-F4: Provide load leveling for pulse power loads such as rail gun PFN, high powered lasers, and advanced radars. Also provide load leveling for PGMs with slow dynamic response such as Fuel Cells. ESMs fulfilling the ESM-F4 function typically have a rating in the100's of $\mathrm{kW}$ to 10 's of MW and a runtime on the order of 5 to 10 seconds. These ESMs typically have a recharge time on the same order of time as the runtime. The ESM-F4 function typically requires a large number of charge-discharge cycles (more than, perhaps much more than 300 per year).

\section{POWER GENERATION AND ENERGY STORAGE IMPLICATIONS}

In designing a shipboard power system, the following guidance should be followed:

a. The total power generation capacity of all PGMs must be at least the worst case load with appropriate margins and service life allowance. For ships with PMMs, this includes the propulsion load.

b. The total power generation capacity of all PGMs less the PGM with the highest power rating must be at least the worst case load with appropriate margins and service life allowance minus the total exempt load for that condition. Note that only ships with PMMs typically have exempt load.

c. For any operating condition / temperature in the Electric Plant Load Analysis (EPLA), the total power generation capacity of all online PGMs and ESMs (fulfilling function ESM-F2) minus the rating of the largest online PGM must be greater than the sum of the online un-interruptible and shortterm interruptible loads. If the power from an ESM can only serve in-zone loads, than any ESM power capacity in excess of the sum of that zone's un-interruptible and short-term interruptible load should not be counted in the total power generation capacity. Fig. 4 shows three representative options for apportioning power ratings among PGMs and ESMs.

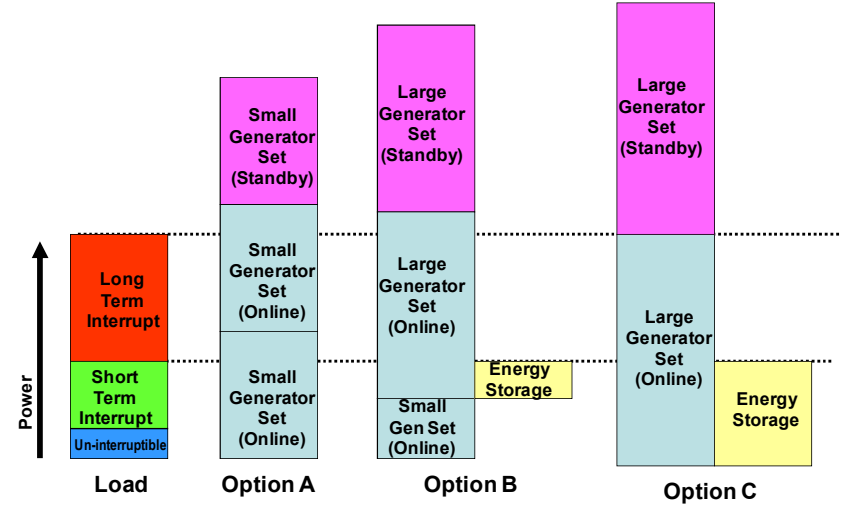

Figure 4. Generator and Energy Storage Sizing d. An ESM fulfilling function $E S M-F 2$ should have a run time of at least 2 to 3 times t 2 to account for multiple power interruptions before the ESM can be recharged. If the recharge time is significantly greater than $\mathrm{t} 2$, then a longer run time of 5 to 6 times $\mathrm{t} 2$ is appropriate.

e. Un-interruptible loads can be served in at least three ways: via a fast power electronics based switch (such as a solid state ABT or a PCM 2A) with two independent sources that are likely not to experience a power outage at the same time (such as when all online PGMs are in parallel); a local ESM fulfilling function ESM-F1 that isolates the uninterruptible load(s) from the power system power outage; or a dedicated UPS. Consideration should be given for integrating the ESM fulfilling function ESM-F1 with the PCM $1 \mathrm{~A}$ or PCM 2A of Fig 2. The dedicated UPS option is likely the most affordable if the number of un-interruptible loads in a zone is minimal. If an UPS is employed, consideration should be given to have it fulfill the $E S M-F 2$ function as well for the loads it serves.

f. The ESMs fulfilling function $E S M-F 3$ should typically be co-located with the PGMs they serve. Consideration should be given to locating an ESM which fulfills both the $E S M-F 2$ and $E S M-F 3$ functions in each space that holds a PGM.

g. If an ESM is required to fulfill the ESM-F4 function to support a PGM, then it should be co-located with the PGM to reduce the probability that the PGM, although functional, would not be able to satisfactorily serve the load because of its inability to dynamically respond to changing load. This same ESM could be employed to serve the pulse power loads. If the $E S M-F 4$ Function is only needed to support the pulse power loads, then consideration should be given to co-locating the ESM with the loads it serves.

It may prove economical to have an ESM fulfilling the $E S M-F 2, E S M-F 3$ and ESM-F4 functions in each space with a PGM. On the other hand, because the ESM-F4 function requires many more charge-discharge cycles, it may be more affordable to split the energy storage requirements into two modules: one ESM sized for the ESM-F4 requirement, but also able to partially fulfill the $E S M-F 2$ and $E S M-F 3$ requirements; and another ESM sized for the remaining ESM$F 2$ and $E S M-F 3$ requirements.

\section{POWER DISTRIBUTION AND CONTROL INTERFACE IMPLICATIONS}

Traditionally, load shedding has been based only on mission priority. Loads were categorized into non-vital, semivital, and vital loads. When a load center detected an underfrequency on the bus, an indicator that load is greater than the generation capacity, then the first stage load shed breaker would trip and de-energize all the non-vital loads. If this is not sufficient for the frequency to recover, then the second stage load shed breaker would trip and de-energize the semivital load. If there still was not sufficient generation, then typically the generator would trip off line on under-frequency. The automatic bus transfer (ABT) for each vital load would sense the loss of power and switch to the alternate power source. The logic presumes that the alternate power source 
has sufficient generation capacity to handle the load added by the ABTs. Fig 5 depicts a representative method for implementing this load shedding strategy in a load center. The circuit breakers with a " $\mathrm{C}$ " imply a circuit breaker that are controlled by the load-shed / machinery control system logic. The remaining circuit breakers trip on over-current or manually.

The traditional load shed strategy does not take into account the load's tolerance of a power interruption. Priority for providing power is solely determined by the importance of the load to the ship's mission. Furthermore, loads requiring un-interruptible power must provide their own Uninterruptible Power Supply.

Power distribution products are now on the market that can implement the control-centric topology depicted in Fig 6. In this topology, all of the breakers can be commanded to open and close by the machinery control system. Un-interruptible loads can be provided with an alternate source of power within the load center as shown, or via an ABT or UPS "downstream" of the load center. With the level of controllability shown in Fig 6, load shedding can initially be based on QOS considerations. As depicted in Fig 7, we would normally expect power to be restored to all loads within time t2 of the loss of a PGM. A service interruption would only occur if multiple failures happened at the same time, or if a single-point-of-failure device fails. As shown in Fig 8, only when the machinery control system determines that sufficient generation cannot be brought online within time $\mathrm{t} 2$, does the load shed strategy shift to one based on mission priority. Some loads that are retained in QOS are shed in order to restore power to other higher mission priority loads.

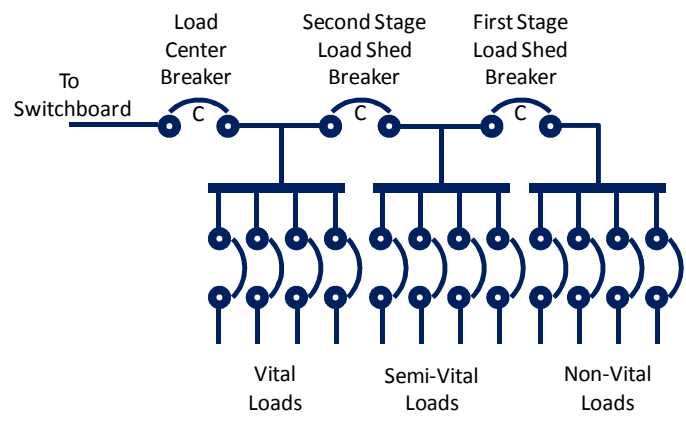

Figure 5. Traditional Load Shedding Implementation in a Load Center

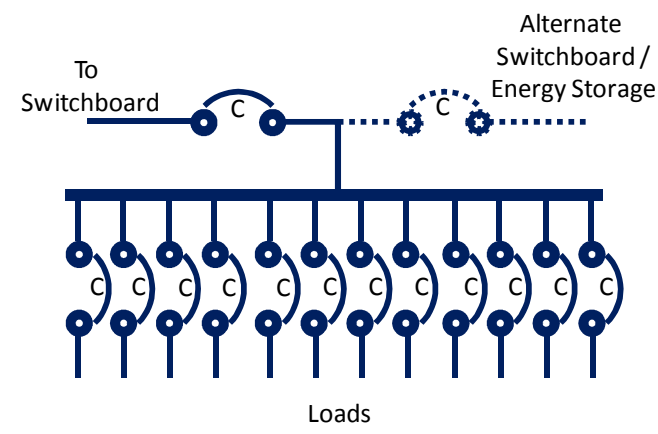

Figure 6. Control centric Load Center topology for implementing QOS and Mission Priority Load Shedding
Within an NGIPS ZEDS implementation, the PCM-1a and PCM-2a described in [5] provide the functionality depicted in Fig 6 with their input and output modules.

In some cases, it may be advantageous and less expensive to implement QOS by modifying the traditional load center implementation as shown in Fig 9. In this example, loads have only one of two mission priorities: Low and High. All un-interruptible loads are presumed to be high priority. With any interruption in power, un-interruptible loads immediately shift to their alternate source, either via an ABT or an alternate source in the load center. If power isn't restored almost immediately, then the High Priority Long Term Interrupt Loads and the Low Priority Long Term Interrupt Loads are shed. If power isn't restored within time t2, then the Low Priority Short Term Interrupt Loads are shed and the High Priority Long Term Interrupt Loads are restored. While this implementation is less flexible than the implementation shown in Fig 6, it may be adequate for some classes of ships.

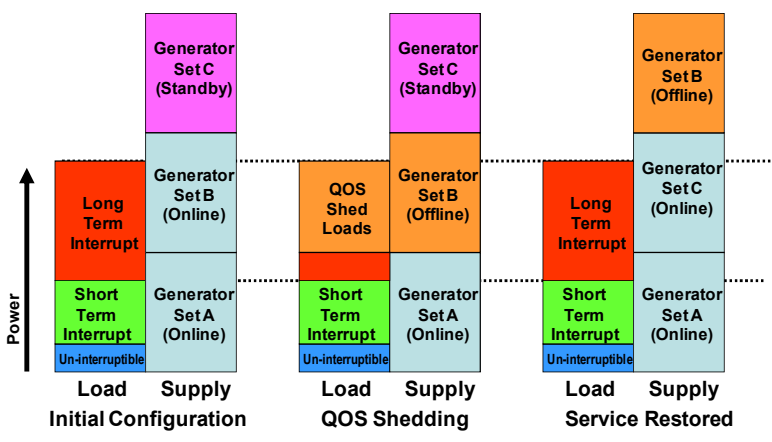

Figure 7. Quality of Service Load Shedding and Restoration of Power

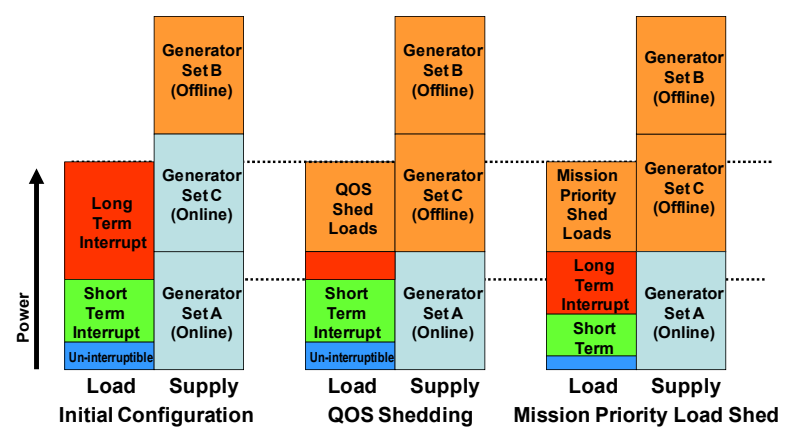

Figure 8. Shift to Mission Priority Load Shed Strategy

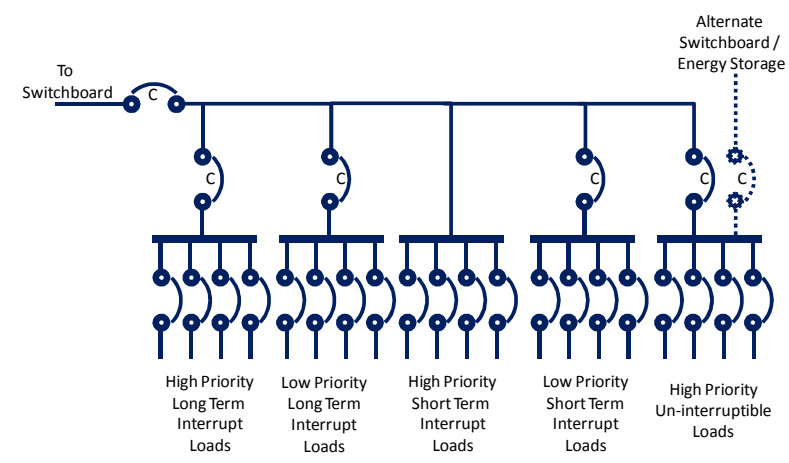

Figure 9. Traditional Load Center implementation modified for QOS 
One potential improvement to Fig 6 is incorporating a robust communications link between the power management software in the machinery control system and the individual loads. With this link, loads could be commanded to minimize power consumption while still staying in a standby mode (or shutdown in an orderly fashion). This could avoid potential equipment failures or improper operation due to a "hard shutdown" of load equipment. The loads may also be able to restore their functionality much quicker once commanded to exit the standby state. This feature could enable substituting a simple breaker for the controlled breaker depicted in Fig 6 . for each load and may result in the re-categorization of a load from an un-interruptible to a short-term interruptible load, or from a short-term interruptible load to a long-term interruptible load. The lack of appropriately adopted open standards for this control and communication link is the principal obstacle to implementing this improvement.

\section{MODELING, SIMULATION, AND ANALYSIS}

One of the challenges in incorporating QOS into a power system design is identifying the amount of long-term interruptible, short-term interruptible and un-interruptible power needed for each operational conditional.

Of first importance to the implementation of a QOS based power system design is establishing MTBSI for the power system design. MTBSI is taken to be an element of the total ship, system of systems, top-level reliability requirement. Hence, it is an independent variable.

Also central to the implementation of a QOS based power system design are:

a. The characterization of all of the loads relative to power system parameters $\mathrm{t} 1$ and $\mathrm{t} 2$, and

b. A tactical scenario dependent mission prioritization of loads.

For the shipboard power systems under consideration here, $\mathrm{t} 1$ and $\mathrm{t} 2$ are typically widely separated, about $60 \mathrm{~s}$, or, two to three possibly four orders of magnitude. Table 1 details the issues of interest for Modeling and Simulation (M\&S) for these different scales of time.

t1 can be on the order of milliseconds, $0.01 \mathrm{~s} \leq \mathrm{t} 1 \leq 2 \mathrm{~s}, \approx 2$ orders of magnitude. The modeling considerations at these time scales are:

- Full order, dynamic, 'switching waveform' load models including controller models

- Full order fault models for the power system

- Tactical scenario models for the ship

- Operator intervention/interaction is not a consideration.

$\mathrm{t} 2$ is on the order of minutes, $60 \mathrm{~s} \leq \mathrm{t} 2 \leq 300 \mathrm{~s}$. The modeling considerations at these time scales are:

- Steady-state, $0^{\text {th }}$, or $1^{\text {st }}$ order load models, generatorset models

- $\quad$ Steady-state, $0^{\text {th }}$, or $1^{\text {st }}$ order operational/campaign/mission models for the ship

- Operator intervention/interaction is meaningful.
Establishing the nominal values of $\mathrm{t} 1$ and $\mathrm{t} 2$ for a power system design requires identification of some of the specific types of equipment to be included in the power system design. $\mathrm{t} 1$ and $\mathrm{t} 2$ are greatly influenced by hardware/equipment.

Assigning ESM functionality on the basis of required MTBSI, $\mathrm{t} 1$ and $\mathrm{t} 2$, and the loads requires significant, detailed information of the power system design and the loads.

QOS Power Management (Load Shedding) and Mission Priority Power Management (Load Shedding) provide the basis for the development of key elements of PCON, a software development.

Modeling and Simulation is an indispensible tool in the design of shipboard power systems, both its hardware and its software. A second consideration in the power system design is that it occurs as an integral part of the total ship design (Fig 10). Herein lies the pacing aspect for the development of the power system model and its utilization. M\&S is an effective design tool when it is focused on answering specific questions. The nature of those questions asked of M\&S dictate which models are most appropriate.

TABLE I. POWER SYSTEM TIME SCALES

\begin{tabular}{|c|c|}
\hline pre t1 & $\begin{array}{c}\text { Isolate the fault. Perform ESM-F1. Perform } \\
\text { ESM-F2. }\end{array}$ \\
\hline t1 & $\begin{array}{c}\text { Reconfigure to supply Short Term Interrupt } \\
\text { loads. Perform ESM-F4 }\end{array}$ \\
\hline t1 to t2 & Ftart additional generator. Perform ESM- \\
& t2 \\
& $\begin{array}{c}\text { Transition from QOS to Mission Priority } \\
\text { Power Management (Load Shedding). }\end{array}$ \\
\hline
\end{tabular}

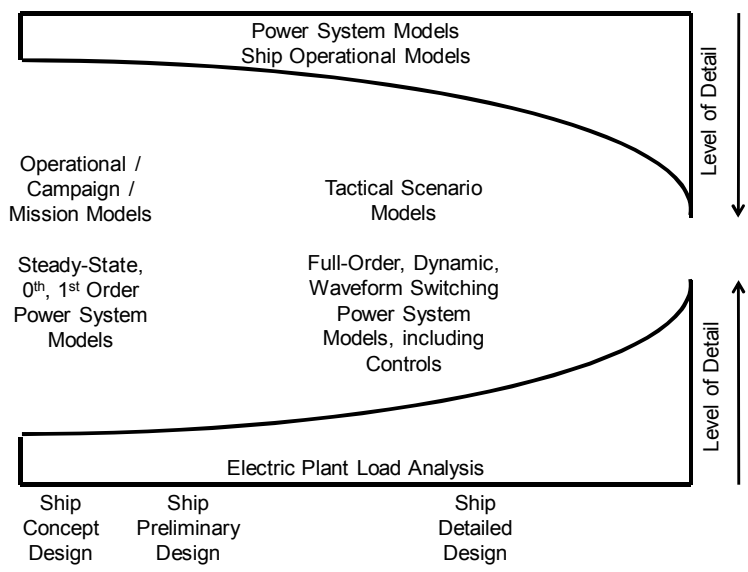

Figure 10. Level of Model Detail vs Ship Design Stage 
In the earlier phases of ship design, top-level decisions must be made, such as the type of prime mover to power the ship. Steady-state, zeroth-, perhaps first- order models of the power system, prime movers for example, are used in the context of top-level ship operational/campaign/mission models to select the type of prime mover. The selection of the type of prime mover is a major influence on $\mathrm{t} 2$, the scale of $E S M-F 3$ and the time constant for resort to Mission Priority Power Management.

In the latter phases of ship design, lower-level decisions must be made, such as design parameters of the electric power distribution system PCMs. Full-order, dynamic, waveform switching models of the power system, the fault isolation behavior, including controls, of the PCM1a for example, are used in the context of ship tactical scenario models to determine whether, for example, fast bus-transfer devices or a greater population of ESM-F1 (ESM-F2) should be implemented in the power system design. The selection between these two is a major influence on $t 1$, the scale of $E S M-F 1$ and ESM-F2 and the time constant for resort to QOS Power Management.

As the ship design progresses through each of its phases, the understanding of the load equipment will increase in scope and detail. For example, in the concept design phase, the EPLA entry for the ship's radar might read, "Missile \& Air Defense Radar - iMW." The model of the radar may be a constant load model. In the detailed design phase, the EPLA entry for the ship's radar might read, "AN/SPSn - j.klMW." The model of the radar may now contain full models of each of the converters between the interface with the power system and the antenna. Such evolution of the population and details of the loads and their models is necessary to be able to assess achievement of the quality of power assumed for the QOS. Such evolution of the population and details of the loads and their models is necessary to develop ESM-F1 and ESM-F4 designs. Such evolution of the population and details of the loads and their models is necessary to develop the QOS and Mission Priority Power Management (Load Shedding) software requirements.

\section{CONCLUSIONS}

Quality of Service plays an important role in ensuring the design of a shipboard power system results in affordable and reliable power continuity as required by each type of load. This paper presented QOS concepts, shown how QOS impacts power system design, and has detailed some of the modeling and simulation considerations.

\section{REFERENCES}

[1] IEEE 1662-2008, "IEEE Guide for the Design and Application of Power Electronics in Electrical Power Systems," Approved 10 November 2008

[2] IEEE 1709-2010, "IEEE Recommended Practice for 1 to $35 \mathrm{kV}$ Medium Voltage DC Power Systems on Ships," Approved June 2010.

[3] CAPT Norbert H.Doerry and David H. Clayton, "Shipboard Electrical Power Quality of Service", 0-7803-9259-0/05/\$20.00 C2005 IEEE, Presented at IEEE Electric Ship Technologies Symposium, Philadelphia, PA, July 25-27, 2005.

[4] CAPT Norbert Doerry, "Designing Electrical Power Systems for Survivability and Quality of Service" presented at ASNE DAY 2007, Arlington, VA, June 25-26, 2007. Also published in ASNE Naval Engineers Journal, 2007, Vol. 119 No 2, pp 25-34.

[5] Norbert Doerry, "NGIPS Technology Development Roadmap " NAVSEA Ser 05D / 349 of 30 Nov 2007

[6] Donald J. Hoffman, Joseph P. Borraccini, Steven Swindler and Captain Lynn Petersen, USN, "Next Generation Power and Energy: Maybe Not so next Generation," Presented at ASNE Engineering the Total Ship (ETS) 2010, July 14-15, 2010, Falls Church, VA.

[7] Figure provided by Electric Ships Office, PMS 320 on Feb 2, 2010. 